\title{
SIMULATION OF SHEET AND CLOUD CAVITATION WITH HOMOGENOUS TRANSPORT MODELS
}

\author{
Bilus, I. ; Morgut, M. $^{* *} \&$ Nobile, E. ${ }^{* *}$ \\ * University of Maribor, Faculty of Mechanical Engineering, Smetanova 17, Maribor, Slovenia \\ ${ }^{* *}$ Università Degli Studi di Trieste, Dipartimento di Ingegneria e Architettura, Via Alfonso Valerio 10, \\ Trieste, Italy \\ E-Mail: ignacijo.bilus@um.si,mmorgut@units.it,nobile@units.it
}

\begin{abstract}
This paper introduces the results of correlated numerical models study carried out to analyse cavitating flows. The flow field of steady attached sheet cavitation and the case of unsteady cavitation behaviour with quasi-periodic fluctuations is analysed with different homogenous cavitation transport models. Three models in form of additional transport equations for water volume fraction are combined with the RANS (Reynolds Averaged Navier-Stokes) equations and calibrated for the cavitating flow around the NACA66 (MOD) hydrofoil by means of an optimisation strategy. In the second stage, the optimised models are applied to the case of internal unsteady cavitating flow in Venturi type section.

The results obtained using calibrated models are very close to each other, and agree well with the available experimental data, indicating that the optimisation process is recommended as a general - first step tool for mathematical models validation.

(Received in July 2012, accepted in February 2013. This paper was with the authors 3 months for 2 revisions.)
\end{abstract}

Key Words: Cavitation, Numerical Simulation, Homogenous Transport Model

\section{INTRODUCTION}

Cavitation is the phenomenon of gas cavities formation inside a liquid medium in low pressure regions, where pressure drops below certain threshold value. Cavitation can cause different undesirable effects such as performance loss, damage by pitting and erosion, structure vibrations and noise generation. Therefore, cavitation process is being investigated intensively in order to prevent the phenomena by all means [1, 2].

Following the fast increase of computer performances in past years, several CFD (Computational Fluid Dynamics) methods were developed to numerically investigate and predict the complicated nature of real flows [3]. The majority of cavitation models are based on the homogeneous fluid approach, where a common flow field and other relevant fields such as temperature and turbulence are shared by both fluids. The variable mixture density can be defined using two common methods. The first is a barotropic law [4] and a second is a transport model for void ratio, with the source terms modelling the mass transfer rate due to cavitation [5-7].

The main purpose of this paper is to evaluate the prediction capabilities of the calibrated mass transfer models for cavitation problems in internal flow. The interphase mass transfer rate due to cavitation is modelled by the source terms implemented in the continuity and liquid volume fraction equations in analysed models. Source terms, known as mass transfer models in this study were computed using the Singhal et al. Full Cavitation Model (FCM) [5], the Zwart et al. model [6] and the Kunz et al. model [7]. For brevity, the models will be indicated as FCM, Zwart and Kunz model in the following. The models share the feature of employing empirical coefficients to guide the evaporation and condensation processes. It 
seems that empirical coefficients cannot have a general character and that default values do not guarantee the accurate simulation results for different cavitating flow regimes.

According to the fact that the considered models involve adjustable parameters, which influence the results accuracy, we considered to properly calibrate the different mass transfer models, and consequently compare them in a possible congruent and fair manner, on a practical flow example. For this purpose, a calibration strategy based on the modeFRONTIER 4.2 optimisation framework [8] was developed.

The present contribution follows the previous numerical optimisation of homogeneous cavitation transport model parameters [9] for the numerical prediction of attached sheet cavitation around a hydrofoil. In previous study, different cavitation transport models were tuned for external, steady flow around hydrofoil NACA66(MOD). At the second stage, the cavitation pattern and pressure coefficient distribution were compared to experimental values for different cavitation coefficient values at different angles of attack [9]. Following the satisfying results of the analysed examples, the same models are here applied to the case of internal flow in the Venturi geometry for the steady [4] and the unsteady flow [10], respectively.

In the following, a description of the mathematical models and then the numerical optimisation strategy is provided, followed by method validation and results comparison.

\section{MATHEMATICAL MODEL}

The governing equations for homogenous cavitation flow modelling using RANS (Reynolds Averaged Navier-Stokes) approach combined with the VOF (Volume of Fluid) method for capturing the liquid-vapour interface are:

- continuity equation:

$$
\boldsymbol{\nabla} \cdot \boldsymbol{U}=\dot{m}\left(\frac{1}{\rho_{l}}-\frac{1}{\rho_{v}}\right)
$$

- momentum equation:

$$
\frac{\partial(\rho \boldsymbol{U})}{\partial t}+\boldsymbol{\nabla} \cdot(\rho \boldsymbol{U} \boldsymbol{U})=-\boldsymbol{\nabla} P+\boldsymbol{\nabla} \cdot \tau+S_{M}
$$

- volume fraction equation:

$$
\frac{\partial \gamma}{\partial t}+\boldsymbol{\nabla} \cdot(\gamma \boldsymbol{U})=\frac{\dot{m}}{\rho_{l}}
$$

Considering the homogenous two phase flow, common flow field and other relevant fields such as temperature and turbulence are shared by both fluids (water and water vapour).

In the equation system above $\boldsymbol{U}[\mathrm{m} / \mathrm{s}]$ represents the time averaged mixture velocity, $P[\mathrm{~Pa}]$ is the time averaged pressure, $\dot{m}[\mathrm{~kg} / \mathrm{s}]$ is the interphase mass transfer rate due to cavitation, $\rho_{v}\left[\mathrm{~kg} / \mathrm{m}^{3}\right]$ is vapour density, $\rho_{l}\left[\mathrm{~kg} / \mathrm{m}^{3}\right]$ is liquid density, $\tau\left[\mathrm{N} / \mathrm{m}^{2}\right]$ is stress tensor, $S_{M}\left[\mathrm{~kg} / \mathrm{m}^{2} \mathrm{~s}^{2}\right]$ are momentum sources and $\gamma$ is water volume fraction. It is defined as:

$$
\gamma=\frac{\text { volume of liquid }}{\text { total volume }}
$$

and related to vapour volume fraction as $\alpha=1-\gamma$ where:

$$
\alpha=\frac{\text { volume of vapour }}{\text { total volume }}
$$

In order to close the set of the governing equations, additional modelling is required to compute the turbulence quantities and the specific interphase mass transfer rate. 
The turbulence quantities were modelled using $k-\varepsilon$ model while interphase mass transfer rate was modelled with three different mass transfer models.

For the purpose of present study, simple linear mixing rule was applied for mixture density and viscosity calculation:

$$
\begin{aligned}
& \rho=\gamma \rho_{l}+(1-\gamma) \rho_{v} \\
& \mu=\gamma \mu_{l}+(1-\gamma) \mu_{v}
\end{aligned}
$$

\section{MASS TRANSFER MODELS}

The mass transfer models define the interphase mass transfer rate caused by cavitation. For the purpose of analysis, three different cavitation transport models were applied. They are formulated as follows.

\subsection{The Singhal model (FCM)}

The mass transfer model proposed by Singhal, originally known as Full Cavitation Model [5], is employed in two well-known commercial CFD codes ANSYS - Fluent and SIMERICS PumpLinx. This model is based on the reduced form of the Rayleigh-Plesset equation for bubble dynamics and its modified formulation [11], where vapour mass fraction is replaced by vapour volume mass fraction. The model states as follows:

$$
\dot{m}= \begin{cases}-C_{e} \frac{\sqrt{k}}{\tau} \rho_{l} \rho_{v} \sqrt{\frac{2}{3} \frac{P_{v}-P}{\rho_{l}}}(1-\alpha) & \text { if } P<P_{v} \\ C_{c} \frac{\sqrt{k}}{\tau} \rho_{l} \rho_{l} \sqrt{\frac{2}{3} \frac{P-P_{v}}{\rho_{l}}} \alpha & \text { if } P>P_{v}\end{cases}
$$

where $k\left[\mathrm{~m}^{2} / \mathrm{s}^{2}\right]$ is turbulence kinetic energy, $\tau[\mathrm{N} / \mathrm{m}]$ is the surface tension, $P_{v}[\mathrm{~Pa}]$ is vapour pressure. $C_{e}$ and $C_{c}$ are empirical coefficients dependent on flow example and operating conditions. Their recommended values are equal $C_{e}=0,02$ and $C_{c}=0,01$.

\subsection{The Zwart model}

The Zwart model is the native ANSYS - CFX mass transfer model. It is based on the simplified Rayleigh-Plesset equation for spherical bubble dynamics. The source terms are defined as follows:

$$
\dot{m}= \begin{cases}-F_{\text {evap }} \frac{3 r_{\text {nuc }}(1-\alpha) \rho_{v}}{R_{B}} \sqrt{\frac{2 \frac{P_{v}-P}{3}}{\rho_{l}}} & \text { if } P<P_{v} \\ F_{\text {cond }} \frac{3 \alpha \rho_{v}}{R_{B}} \sqrt{\frac{2}{3} \frac{P-P_{v}}{\rho_{l}}} & \text { if } P>P_{v}\end{cases}
$$

In the above equations, $r_{\text {nuc }}$, is the nucleation site volume fraction, $R_{B}[\mathrm{~m}]$ is the radius of a nucleation site, $F_{\text {evap }}$ and $F_{\text {cond }}$ are two empirical calibration coefficients. In CFX the above mentioned coefficients, by default, are set as follow: $r_{n u c}=5 \cdot 10^{-4}, R_{B}=2 \cdot 10^{-6} \mathrm{~m}$, $F_{\text {evap }}=50, F_{\text {cond }}=0,01$.

It is evident from the model above, that expressions are not symmetric for the case of the evaporation and condensation, respectively. The author replaced the $\alpha$ by $r_{n u c}(1-\alpha)$ in the 
evaporation term in order to take in the account that nucleation site density decreases when vapour volume fraction increases.

\subsection{The Kunz model}

The Kunz cavitation transport model is heuristic model based on the work by Merkle [12] and one of the mass transfer models implemented in open source Open-FOAM CFD software package, which is frequently used in many heat $[13,14]$ and fluid $[15,16]$ flow analysis.

In Kunz model, unlike the above mentioned model, the mass transfer is based on two different strategies for creation $\dot{m}^{+}$and destruction $\dot{m}^{-}$of liquid. The transformation of liquid to vapour is calculated as being proportional to amount by which the pressure is below the vapour pressure. The transformation of vapour to liquid, otherwise, is based on the third order polynomial function of volume fraction, $\gamma$. The specific mass transfer rate is defined as $\dot{m}=\dot{m}^{+}+\dot{m}^{-}$:

$$
\dot{m}= \begin{cases}\frac{C_{\text {prod }} \rho_{v}\left(\gamma^{2}-\gamma^{3}\right)}{t_{\infty}} & \text { if } P<P_{v} \\ \frac{C_{d e s t} \rho_{v} \gamma \min \left(0, P-P_{v}\right)}{\left(\frac{1}{2} \rho_{l} U_{\infty}^{2}\right) t_{\infty}} & \text { if } P>P_{v}\end{cases}
$$

In the above equations $U_{\infty}[\mathrm{m} / \mathrm{s}]$ is the freestream velocity, $t_{\infty}[\mathrm{s}]=l / U_{\infty}$ is the mean flow time scale, where $l[\mathrm{~m}]$ is the characteristic length scale. $C_{\text {dest }}$ and $C_{\text {prod }}$ are two empirical coefficients. In the original formulation $C_{\text {dest }}=100, C_{\text {prod }}=100$.

For all models presented above, the maximum density ratio was limited to 1000 by the numerical scheme. The limitation was assumed to be properly chosen in order to guarantee the best convergence and robustness of the solver.

\section{THE CALIBRATION PROCESS}

In analysed mass transfer models, different empirical values are present. They directly influence the evaporation and the condensation processes and therefore the mass transfer. This means that the accuracy of the individual mass transfer model is strictly related to the proper prescription of its empirical values.

To properly tune the different mass transfer models, and consequently to compare and use them, a calibration strategy based on the modeFRONTIER 4.2 optimisation system was developed and presented in [9]. The optimisation results are summarised in the table below.

Table I: The optimisation results.

\begin{tabular}{|l|c|c|c|c|c|c|}
\hline \multirow{2}{*}{$\begin{array}{c}\text { Coefficient } \\
\text { value }\end{array}$} & \multicolumn{2}{|c|}{ Zwart } & \multicolumn{2}{c|}{ Singhal (FCM) } & \multicolumn{2}{c|}{ Kunz } \\
\cline { 2 - 7 } & $F_{\text {evap }}$ & $F_{\text {cond }}$ & $C_{e}$ & $C_{c}$ & $C_{\text {dest }}$ & $C_{\text {prod }}$ \\
\hline Default & 50 & 0.01 & 0.02 & 0.01 & 100 & 100 \\
\hline Optimised & 300 & 0.03 & 0.4 & $2.3 \times 10^{-4}$ & 4100 & 455 \\
\hline
\end{tabular}

\section{RESULTS VALIDATION ON THE VENTURI SECTION}

Following the satisfactory results for the hydrofoil [9] where good agreement was found between prediction and measurements and no significant differences were observed between the different models, the mass transfer models were applied to the Venturi section geometries. For the purpose of steady flow study, the Venturi type test section geometry used by Barre [4] 
was adopted in the first step. In order to analyse the reliability of models for the transient flow example, the models were applied to the unsteady Venturi case, where quasi-periodic fluctuations are reported from experiment [10].

\subsection{Steady Venturi section geometry}

The Venturi test section consisted in parallel sidewalls generating rectangular cross section. The profile with a convergence angle of $4.3^{\circ}$ and divergence angle of $4^{\circ}$ was used. To fix the separation point of cavitation cloud, the edge, forming the throat of height $43.7 \mathrm{~mm}$, was used (Fig. 1).

For the case of the Kunz transport model the domain length was used as a length scale $L$.

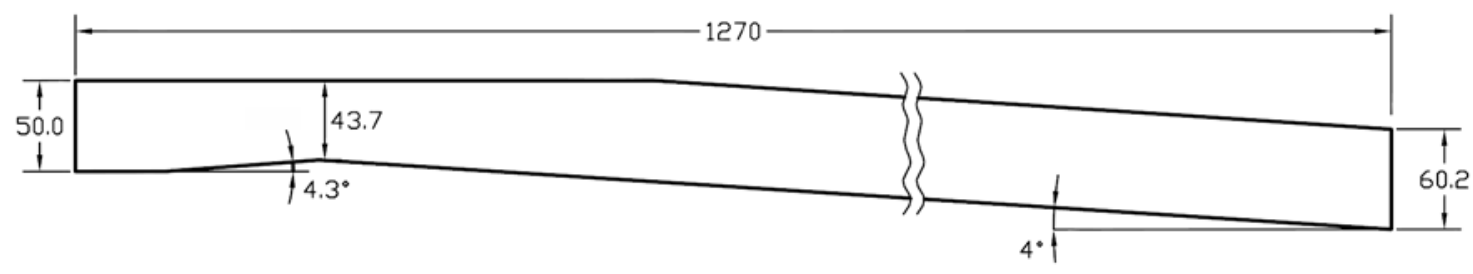

Figure 1: Schematic view of Venturi section (Barre).

The operating point of the system was defined by the following boundary conditions in Table II (values for steady Venturi section geometry).

Table II: Boundary conditions for steady and unsteady Venturi section geometry.

\begin{tabular}{|c|c|c|c|}
\hline Boundary & Variable & Value (steady) & Value (unsteady) \\
\hline Inlet & velocity & $12.2 \mathrm{~m} / \mathrm{s}$ & $7.2 \mathrm{~m} / \mathrm{s}$ \\
\hline Outlet & relative static pressure & $101325 \mathrm{~Pa}$ & $101325 \mathrm{~Pa}$ \\
\hline Top, bottom & wall & no-slip & no-slip \\
\hline Sidewalls & symmetry condition & - & - \\
\hline
\end{tabular}

Water and vapour volume fractions were set equal to 1 and 0 at the inlet boundary, respectively. The cavitating flow regimes related to the cavitation number, $\sigma=0,59$, and $\sigma=0,64$ defined the value of the saturation pressure $P_{v}$.

\subsection{Unsteady Venturi section geometry}

For the purpose of the numerical analysis of successive steps of cavity cycle, the Venturi type test section geometry presented by Coutier-Delgoscha [10] was adopted.

The Venturi test section consisted in parallel sidewalls generating rectangular cross section. The profile with a convergence angle of $17^{\circ}$ and divergence angle of $7^{\circ}$ was used. To fix the separation point of cavitation cloud, the edge, forming the throat of height $33.5 \mathrm{~mm}$, was used (Fig. 2).

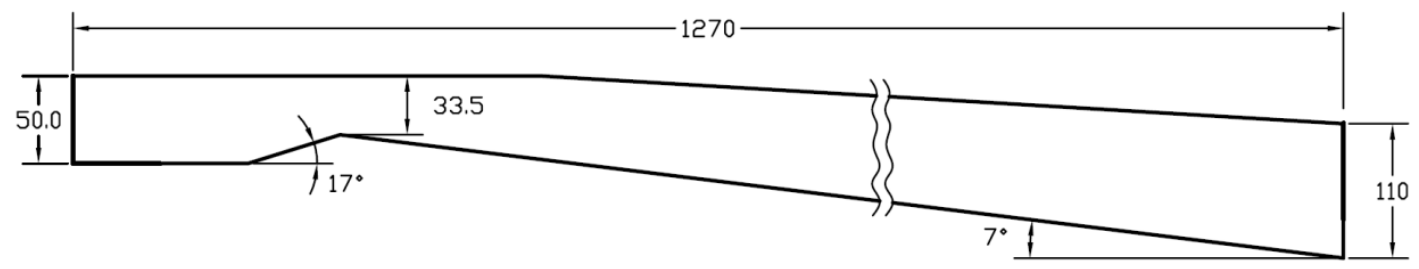

Figure 2: Schematic view of Venturi section (Coutier-Delgoscha). 
The operating point of the system was defined by following boundary conditions in Table II (values for unsteady Venturi section geometry).

Water and vapour volume fractions were set equal to 1 and 0 at the inlet, respectively. The cavitating flow regimes related to the cavitation number, $\sigma=2,4$ and $\sigma=2,44$ defined the value of the saturation pressure $P_{v}$. Timestep was $\Delta t=0.001 \mathrm{~s}$ and total simulation time was $30 \cdot T_{\text {ref }}$, where $T_{\text {ref }}$ was defined as:

$$
T_{\text {ref }}=\frac{\text { chord of the Venturi }}{U_{\infty}}
$$

\subsection{Mesh independence study}

It should be one of the goals of each numerical analysis a comprehensive evaluation of CFD software used for particular application. Such an evaluation should ensure that different types of errors are identified, treated separately and reduced bellow an acceptable level [15].

In the present work, the influence of computational grid refinement on numerical results was studied throughout the examination of spatial (grid) convergence using three hexa structured meshes. The mesh independence study for the steady flow example is summarised in Table III.

Table III: The mesh independence study results.

\begin{tabular}{|l|c|c|c|c|}
\hline \multirow{2}{*}{ Mesh } & \multirow{2}{*}{$\begin{array}{c}\text { Number } \\
\text { of nodes }\end{array}$} & Zwart & Singhal (FCM) & Kunz \\
\cline { 3 - 5 } & 16520 & $p_{\text {inlet }}$ & $p_{\text {inlet }}$ & $p_{\text {inlet }}$ \\
\hline Coarse & 92292.4 & 89451 & 92037.6 \\
\hline Medium & 61040 & 92989.6 & 92776.4 & 92694.8 \\
\hline Fine & 141200 & 92945 & 92918.3 & 92758.3 \\
\hline
\end{tabular}

Following the results of this analysis, the discretisation uncertainty was calculated using procedure introduced by Celik et al. [17]. The maximum calculated uncertainty was less than $1 \%$ for all mesh sizes. Accounting for numerical accuracy and number of elements, the medium mesh was used for further analysis.

It should be noted, that, due to the non-uniform grid refinement, this is an iterative procedure, which, in the case of uniform grid refinement (mainly encountered in 2dimensional numerical analyses of fluid $[16,18]$ and heat $[19,20]$ flow), simplifies to a straightforward numerical accuracy assessment.

\subsection{Steady simulation results}

The simulation results for the steady Venturi case are shown in Fig. 3.

The results show the signification of calibration process introduced. It is evident, that optimisation assures more acceptable results than default values in the case of Singhal (FCM) and Kunz models. In fact, the default coefficient values seem not to catch the cavitation regime in the case when the Kunz model is used. 


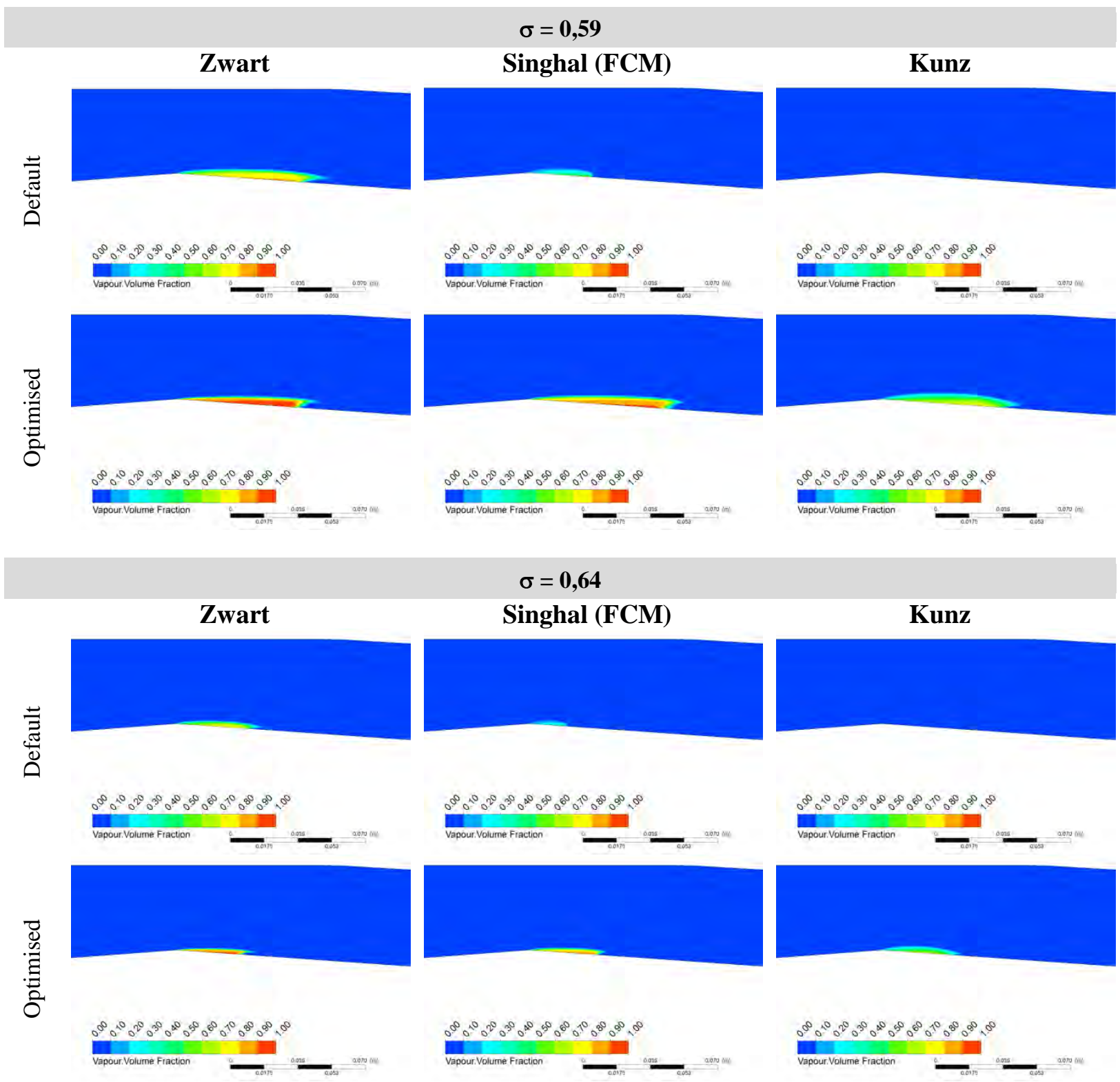

Figure 3: Cavitation in the Venturi section for $\sigma=0,59$ and $\sigma=0,64$.

Table IV: The cavitation sheet lengths.

\begin{tabular}{|c|c|c|c|}
\hline $\boldsymbol{\sigma}=\mathbf{0 , 5 9}$ & Zwart & Singhal (FCM) & Kunz \\
\hline Default & $l=79 \mathrm{~mm}$ & $l=32 \mathrm{~mm}$ & no cavitation \\
\hline Optimised & $l=74 \mathrm{~mm}$ & $l=73 \mathrm{~mm}$ & $l=72 \mathrm{~mm}$ \\
\hline
\end{tabular}

\begin{tabular}{|c|c|c|c|}
\hline $\boldsymbol{\sigma}=\mathbf{0 , 6 4}$ & Zwart & Singhal (FCM) & Kunz \\
\hline Default & $l=40 \mathrm{~mm}$ & $l=17 \mathrm{~mm}$ & no cavitation \\
\hline Optimised & $l=36 \mathrm{~mm}$ & $l=37 \mathrm{~mm}$ & $l=37 \mathrm{~mm}$ \\
\hline
\end{tabular}

Comparing the cavity length for $\sigma=0,59$, it can be observed that the Zwart model with cavity length $l=74 \mathrm{~mm}$, Singhal model with cavity length $l=73 \mathrm{~mm}$ and the Kunz model with cavity length $l=72 \mathrm{~mm}$ agree well with the experimental values reported by Barre 
(70 $<l<85 \mathrm{~mm}$ ). Comparing the cavity lengths for $\sigma=0,64$ and $\sigma=0,59$ the reasonable decrease is evident. It is also evident, for both sigmas analysed, that the Zwart model, which is the default cavitation model in ANSYS CFX, in default version seems to be the most appropriate model for simulation performed and that the Kunz model constants should be optimised for cavitation simulation in any case.

Fig. 4 shows a comparison of the spatial evolution of the longitudinal velocity at two regions. The local speed in the initial part of the sheet is compared at $X_{1}=5,1 \mathrm{~mm}$ and $X_{2}=20,9 \mathrm{~mm}$ from the throat of the Venturi and in the rear part of the sheet at $X_{3}=38,4 \mathrm{~mm}$ and $X_{4}=73,9 \mathrm{~mm}$ from the throat.

The diagrams show the experimental values [4] in terms of most probable velocity $\mathrm{v}_{\mathrm{mp}}$ determined using Probability Density Function (PDF) and numerical values obtained using different mass transfer models for the case of $\sigma=0,59$.

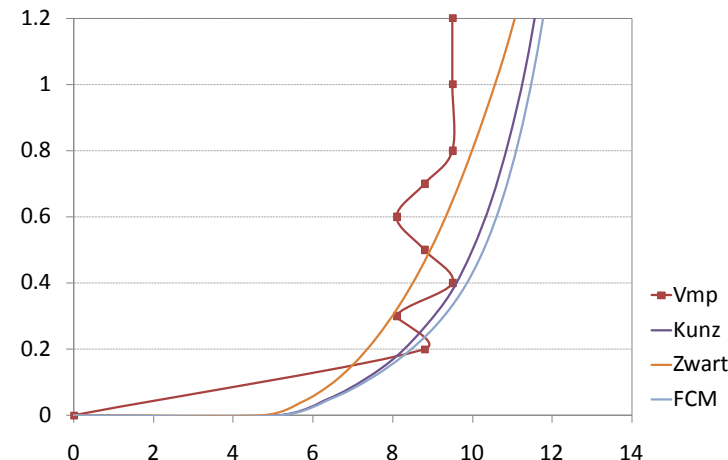

a)

$$
X_{1}=5,1 \mathrm{~mm}
$$

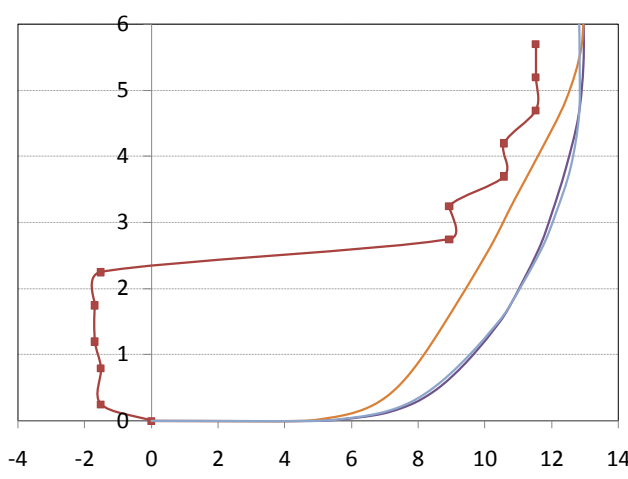

c)

$$
X_{3}=38,4 \mathrm{~mm}
$$

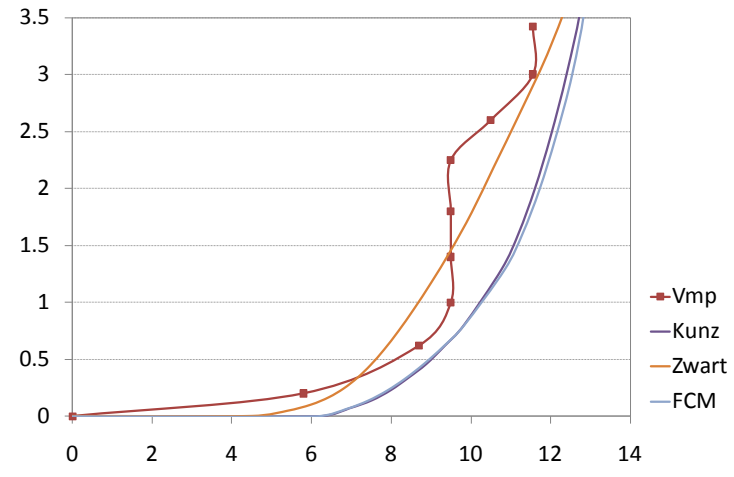

b)

$$
X_{2}=20,9 \mathrm{~mm}
$$

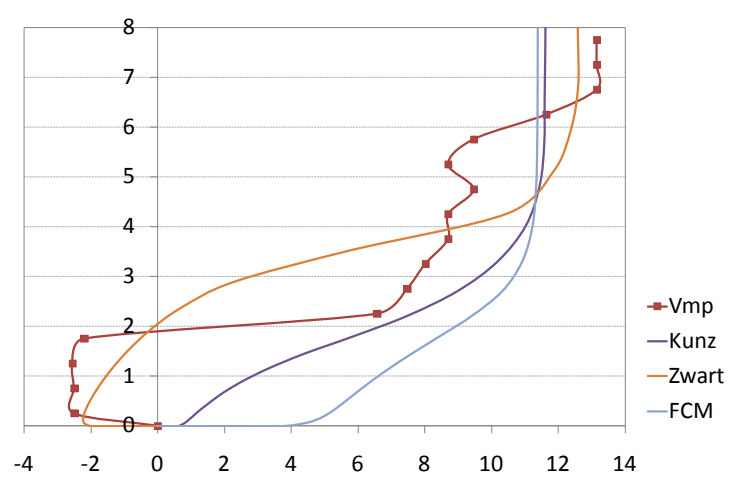

d)

$$
X_{4}=73,9 \mathrm{~mm}
$$

Figure 4: Velocity profiles comparison at initial and rear part of the sheet.

Figs. 4a and $4 \mathrm{~b}$ show experimental velocity profile close to a turbulent boundary layer type near the wall. However, the velocity profiles obtained by numerical simulations are qualitatively comparable to experiment at initial part of the sheet. It seems that velocity profile calculated using the Zwart model fits the experiment best as also expected referring to Fig. 5.

On the rear part of the sheet (Figs. 4c and 4d), a re-entrant jet formation is visible from the $v_{m p}$ profile. At the distance $X_{3}=38,4 \mathrm{~mm}$ from the throat, numerical results show the boundary layer type profiles which do not match the experiment. At the $X_{4}=73,9 \mathrm{~mm}$ numerical results, and the Zwart model in particular, predict the re-entrant jet structure, but the re-entrant jet thickness is not following the experimental profiles. 


\subsection{Unsteady simulation results}

The simulation results for the unsteady Venturi case are shown in Fig. 5. The figure shows the transient evolution of cavitating flow at $\sigma=2,44$ for all constitutive models, since the final cavity obtained is fundamentally unstable.

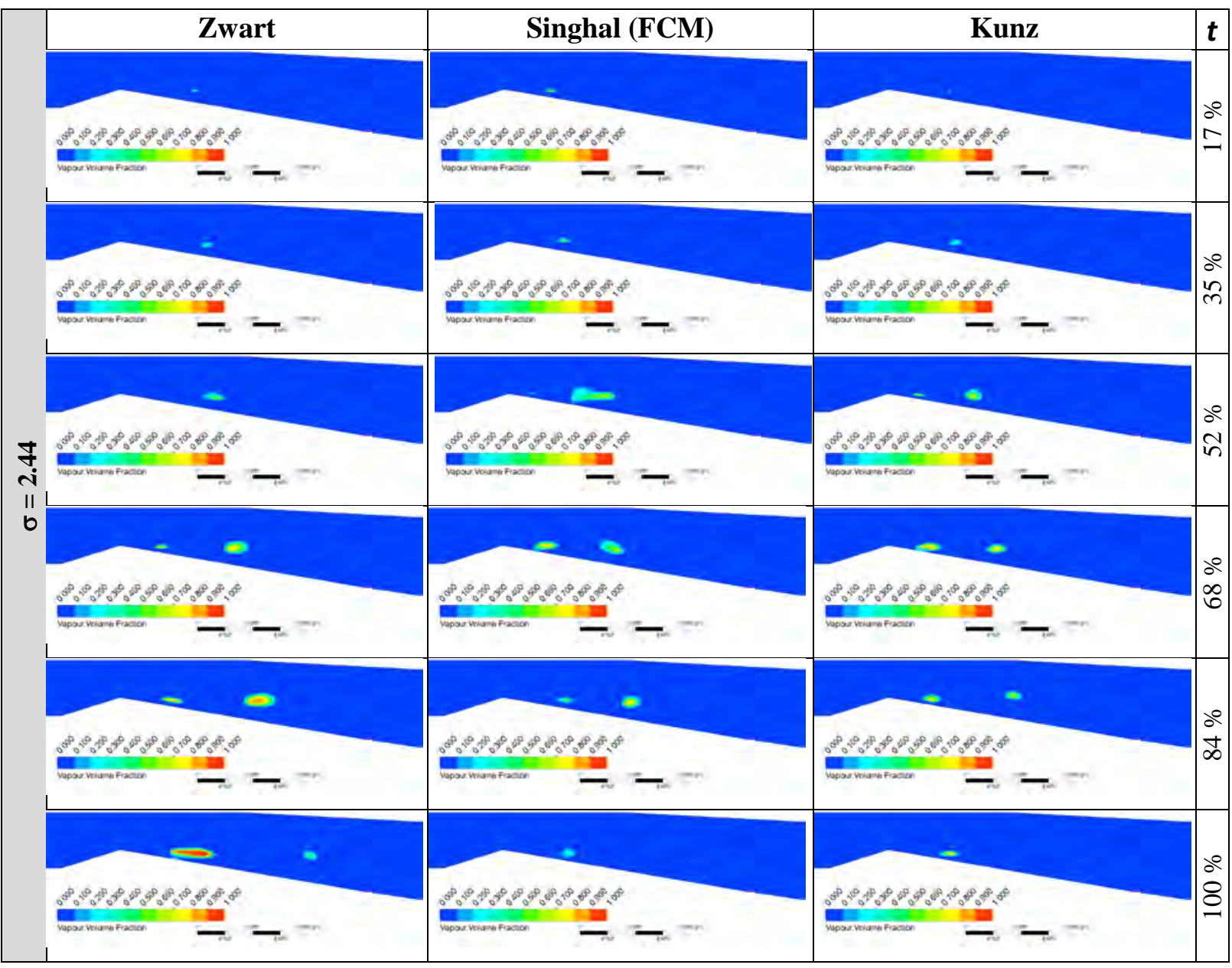

Figure 5: Behaviour of cavitation cloud for $\sigma=2,44$.

It is evident from Fig. 5 that the models results are reasonably in line with each other. The distance from the Venturi throat which defines the maximum cloud length was in the range 120-140 mm. In order to analyse more intensive cavitation regimes the simulation was performed for the value of $\sigma=2,40$. The results are visualized in Fig. 6 .

It is evident from Fig. 6, that the cavitation cloud cycle is comparable when using optimised (calibrated) models. The calculated length of cavitation zone is up to $260 \mathrm{~mm}$.

Following the fact that comparisons are based on transient evolution of cavitating cloud and therefore based on cavitation volume, shape and length further quantitative conclusions were searched. It is well known that cloud cavitation has a periodic character, which is related to process of shedding and collapse of cavitation bubbles. Therefore the periodicity and the cavitation cycle frequency was determined using FFT analysis of the inlet pressure. FFT analysis shows dominating frequencies in the range below $50 \mathrm{~Hz}$. The major pressure pulsations frequency increases with cavitation number. 


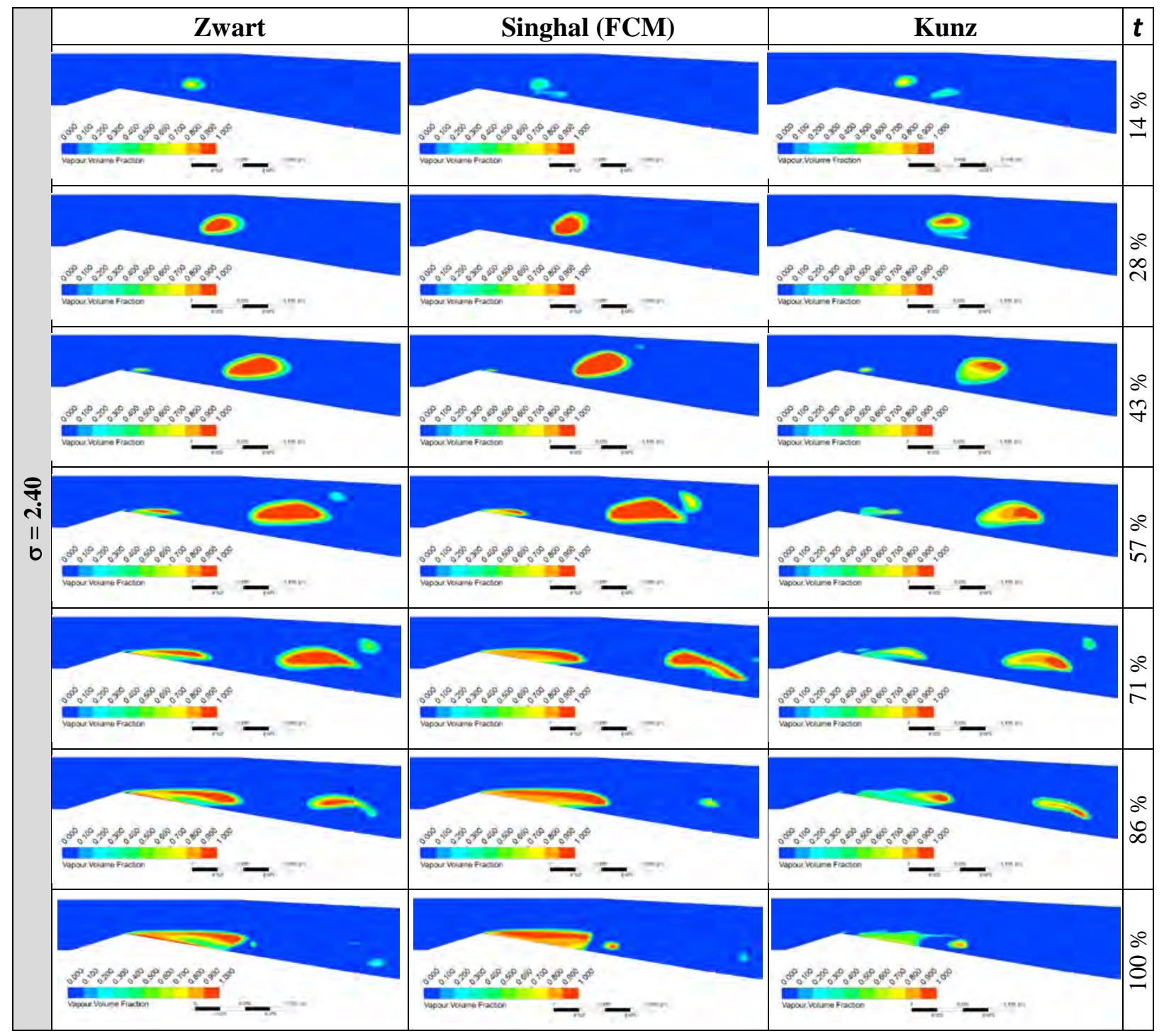

Figure 6: Behaviour of cavitation cloud for $\sigma=2,40$.

Table V: The oscillation frequencies.

\begin{tabular}{|c|c|c|c|c|}
\hline \multirow{2}{*}{$\sigma$} & \multicolumn{4}{|c|}{ Oscillation frequency [Hz] } \\
\cline { 2 - 5 } & Zwart & Singhal (FCM) & Kunz & Delgoscha \\
\hline 2.44 & 44.9 & 44.1 & 44.8 & 68 \\
\hline 2.40 & 19.0 & 19.0 & 21.9 & 55 \\
\hline
\end{tabular}

Although the qualitative behaviour was correctly reproduced, the frequencies calculated differ from the results presented by Delgoscha [10]. The reason for this could be in the differences of physical models applied and simplifications made.

Apart from the differences between the variable analysed, the limitations of the used numerical models have to be taken into account also. The fact is, that the transport equation models presented do not capture the phenomena of large eddies and vorticity production connected to implosion phase of cavitation cloud, Gopalan and Katz [21]. According to this further analyse and optimisation of models is required. 


\section{DISCUSSION}

Notwithstanding the discrepancies observed in velocity profile distributions at cavitation intensive operating regimes, for the case of steady simulation of attached sheet cavitation, homogenous cavitation models results based on the additional transport equation are comparable. Moreover, the results of simulations based on the optimised transport models seem to qualitatively follow the trends of the re-entrant jet formation at the trailing edge of the sheet.

The models, despite the fact of being optimised, only marginally follow the physics when analysing the unsteady regime, where liquid-vapour interaction (in the region of collapsing cloud) causes significant change of momentum and turbulence quantities.

The optimisation results show the uniformity of approach for all analysed models. According to this, the optimisation strategy which defines the empirical values of the source terms, without regard to mathematical model, seems to be of crucial importance for simulation accuracy for the cases where the phenomena could be analysed in the steady frame of reference.

In order to extend the value of the presented strategy to additional unsteady cavitation aspects and pulsating nature of multiphase mixture [22], further work is required to include the influence of time dependent behaviour during cavitation lifecycle on the numerical models in the segment of momentum equation and turbulence models.

\section{CONCLUSION}

Continuous increase of computer capabilities, intensive experimental methods development caused CFD to become a useful tool at the scientific and engineering field of multiphase fluid flow studies. In the case of cavitation, several homogenous transport models based on RANS equations, VOF approach and transport equation for void ratio are presented in open literature.

These transport equations are derived following different mathematical procedures and developed for different flow examples. For this reason they include different source terms definitions and should be properly calibrated. According to this, the calibration procedure based on the modeFRONTIER optimization framework was applied to three cavitation transport models for the case of external flow around NACA 66MOD. In the next step, the optimisation strategy results were used for simulation of steady and unsteady flow in a Venturi nozzle.

The results show that proper condensation and evaporation parameters prescription influence the results accuracy in models discussed.

The results show that optimised cavitation transport models can be used for qualitative analyses of the steady sheet cavitation in the Venturi nozzle. For the case of unsteady sheet and sheet-cloud cavitation, further work is required on the models.

It is recommended to use the optimisation process every time when transport model is validated. Afterwards, optimized model can be applied to physically similar examples for external and internal flows regarding to cavitation coefficient values.

Finally, it was observed that the differences between the numerical and experimental results increase with the extensiveness of the cavitation. The reason for this should be found in raised level of instabilities connected to intensive liquid - vapour interaction and limitations applied with steady simulation approach and homogenisation of two phase flow. 


\section{REFERENCES}

[1] Jost, D.; Lipej, A. (2011). Numerical prediction of non-cavitating and cavitating vortex rope in a Francis turbine draft tube, Strojniski vestnik - Journal of Mechanical Engineering, Vol. 57, No. 6, 445-456, doi:10.5545/sv-jme.2010.068

[2] Gregorc, B.; Predin, A.; Fabijan, D.; Klasinc, R. (2012). Experimental analysis of the impact of particles on the cavitating flow, Strojniski vestnik - Journal of Mechanical Engineering, Vol. 58, No. 4, 238-244, doi:10.5545/sv-jme.2011.062

[3] Stupar, S.; Isakovic, J.; Komarov, D.; Simonovic, A.; Damljanovic, D. (2013). Computational and experimental investigation into subsonic flow around a finned ogive cylinder, Transactions of FAMENA, Vol. 36, No. 4, 97-110

[4] Barre, S.; Rolland, J.; Boitel, G.; Goncalves, E.; Fortes-Patella, R. (2009). Experiments and modelling of cavitating flows in Venturi: Attached sheet cavitation, European Journal of Mechanics B/Fluids, Vol. 28, No. 3, 444-464, doi:10.1016/j.euromechflu.2008.09.001

[5] Singhal, A. K.; Athavale, M. M.; Li, H.; Jiang, Y. (2002). Mathematical basis and validation of the full cavitation model, Journal of Fluids Engineering, Vol. 124, No. 3, 617-624, doi:10.1115/1.1486223

[6] Zwart, P. J.; Gerber, A. G.; Belamri, T. (2004). A two-phase flow model for predicting cavitation dynamics, ICMF-2004 International Conference on Multiphase Flow, Paper No. 152, 11 pages

[7] Kunz, R. F.; Boger, D. A.; Stinebring, D. R.; Chyczewsky, T. S.; Lindau, J. W.; Gibeling, H. J.; Venkateswaran, S.; Govindan, T. R. (2000). A preconditioned Navier-Stokes method for twophase flows with application to cavitation prediction, Computers and Fluids, Vol. 29, No. 8, 849875, doi:10.1016/S0045-7930(99)00039-0

[8] ESTECO srl (1999-2009). modeFRONTIER 4.2, Users Manual

[9] Morgut, M.; Nobile, E.; Bilus, I. (2011). Comparison of mass transfer models for the numerical prediction of sheet cavitation around a hydrofoil, International Journal of Multiphase Flow, Vol. 37, No. 6, 620-626, doi:10.1016/j.ijmultiphaseflow.2011.03.005

[10] Delgoscha, O. C.; Fortes-Patella, R.; Reboud, J.-L.; Stutz, B. (2004). Test case No. 30: Unsteady cavitation in a Venturi type section, Multiphase Science and Technology, Vol. 16, No. 1-3, 207218, doi:10.1615/MultScienTechn.v16.i1-3.290

[11] Huuva, T. (2008). Large eddy simulation of cavitating and noncavitating flow, Ph.D. Thesis, Department of Shipping and Marine Technology, Chalmers University of Technology, Gothenburg, Sweden

[12] Merkle, C. L.; Feng, J.; Buelow, P. E. O. (1998). Computational modelling of the dynamics of sheet cavitation, Proceedings of the $3^{\text {rd }}$ International Symposium on Cavitation, Grenoble, France

[13] Ternik, P.; Rudolf, R. (2012). Heat transfer enhancement for natural convection flow of waterbased nanofluids in a square enclosure, International Journal of Simulation Modelling, Vol. 11, No. 1, 29-39, doi:10.2507/IJSIMM11(1)3.198

[14] Ternik, P.; Rudolf, R.; Zunic, Z. (2012). Numerical study of heat transfer enhancement of homogeneous water-Au nanofluid under natural convection, Materials and Technology, Vol. 46, No. 3, 257-261

[15] Ternik, P. (2010). New contributions on laminar flow of inelastic non-Newtonian fluid in the two-dimensional symmetric expansion: Creeping and slowly moving flow conditions, Journal of Non-Newtonian Fluid Mechanics, Vol. 165, No. 19-20, 1400-1411, doi:10.1016/j.jnnfm.2010.07. $\underline{007}$

[16] Bilus, I.; Ternik, P.; Zunic, Z. (2011). Further contributions on the flow past a stationary and confined cylinder: Creeping and slowly moving flow of Power law fluids, Journal of Fluids and Structures, Vol. 27, No. 8, 1278-1295, doi:10.1016/j.jfluidstructs.2011.06.004

[17] Celik, I. B.; Ghia, U.; Roache, P. J.; Freitas, C. J. (2008). Procedure for estimation and reporting of uncertainty due to discretization in CFD applications, Journal of Fluids Engineering, Vol. 130, No. 7, 4 pages, doi:10.1115/1.2960953

[18] Ternik, P. (2009). Planar sudden symmetric expansion flows and bifurcation phenomena of purely viscous shear-thinning fluids, Journal of Non-Newtonian Fluid Mechanics, Vol. 157, No. 1-2, 15-25, doi:10.1016/j.jnnfm.2008.09.002 
[19] Ternik, P.; Rudolf, R.; Zunic, Z. (2013). Numerical study of Rayleigh-Benard natural convection heat-transfer characteristics of water-based Au nanofluids, Materials and Technology, Vol. 47, No. 2, 211-215

[20] Ternik, P.; Rudolf, R. (2013). Laminar natural convection of non-Newtonian nanofluids in a square enclosure with differentially heated side walls, International Journal of Simulation Modelling, Vol. 12, No. 1, 5-16, doi:10.2507/IJSIMM12(1)1.215

[21] Gopalan, S.; Katz, J. (2000). Flow structure modelling issues in the closure region of attached cavitation, Physics of Fluids, Vol. 12, No. 4, 895-911, doi:10.1063/1.870344

[22] Foldyna, J.; Klich, J; Hlavacek, P.; Zelenak, M.; Scucka, J. (2012). Erosion of metals by pulsating water jet, Technical Gazette, Vol. 19, No. 2, 381-386 